Document downloaded from:

http://hdl.handle.net/10251/102267

This paper must be cited as:

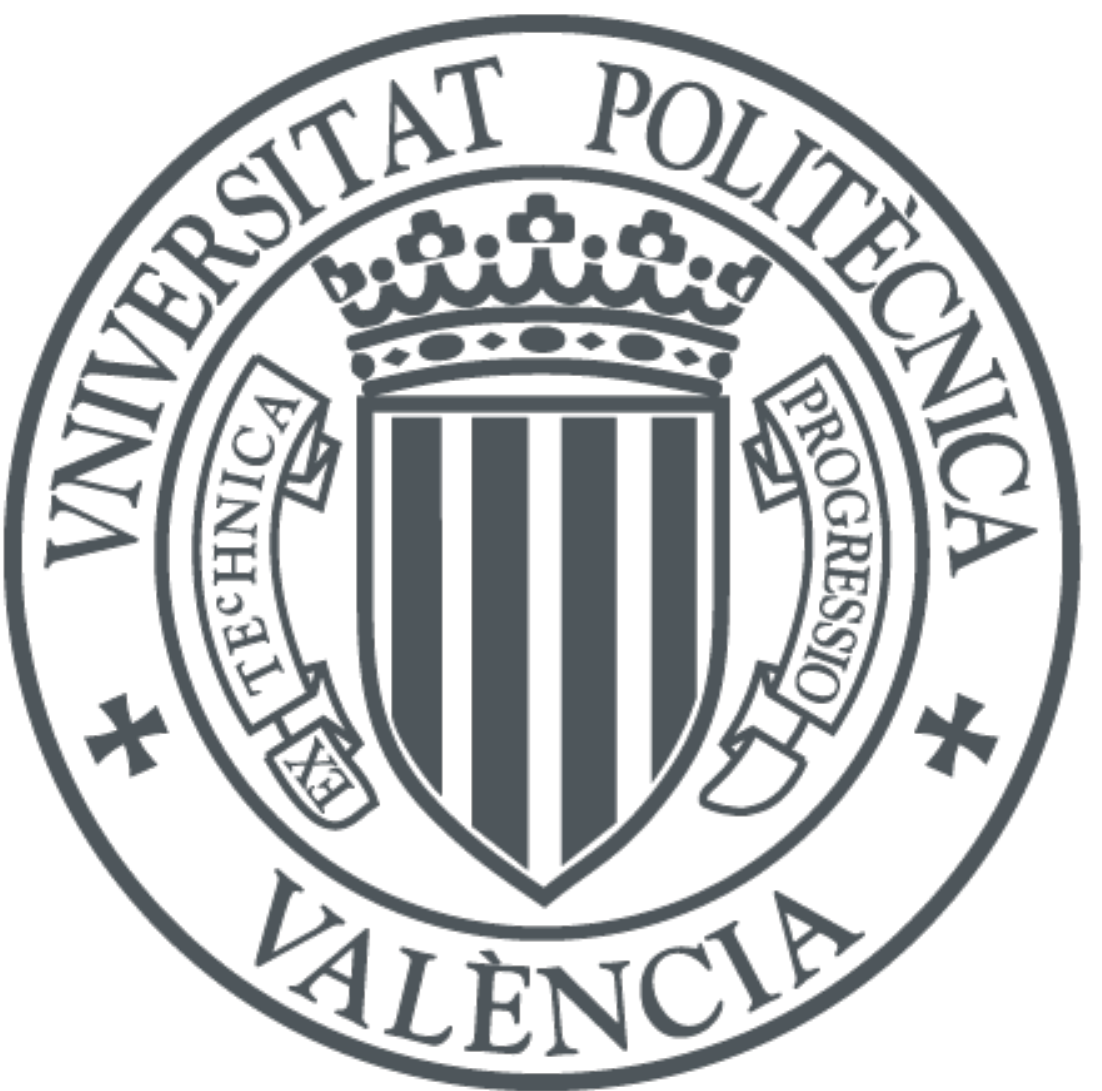

The final publication is available at

https://doi.org/10.1016/j.nucengdes.2017.04.037

Copyright Elsevier

Additional Information 


\title{
Uncertainty Analysis of PKL SBLOCA G7.1 Test Simulation using TRACE with Wilks and GAM Surrogate Methods
}

\author{
F. Sanchez-Saez ${ }^{\mathrm{a}, *}$, S. Carlos ${ }^{\mathrm{a}}$, J.F. Villanueva ${ }^{\mathrm{a}}$, A.I. Sanchez ${ }^{\mathrm{b}}$, S. Martorell ${ }^{\mathrm{a}}$ \\ ${ }^{a}$ Departament d'Enginyeria Química i Nuclear, Universitat Politècnica de València. Camí de Vera, 14. València. Spain. \\ ${ }^{b}$ Departament d'Estadística i Investigació Operativa, Universitat Politècnica de València. Camí de Vera, 14. València. \\ Spain.
}

\begin{abstract}
The Nuclear Energy Agency auspices simulation of experiments in different facilities under several programs. One on them consisted of performing a counterpart test between ROSA/LSTF and PKL facilities, with the main objective of determining the effectiveness of late accident management actions in a small break loss of coolant accident. The results obtained by TRACE code for PKL experiment SBLOCA G7.1 (a scaled model of Konvoi reactor) were in good agreement with the experiments. However, in the simulation process, uncertainty was not accounted. Uncertainty analysis, following the principles of Best Estimate Plus Uncertainty (BEPU) approach, must be performed to measure the effect of uncertainties on the evolution of safety variables of interest, such as the maximum of the Peak Cladding Temperature (PCTmax) in the experiment. In this paper we present a comparison between two uncertainty analysis techniques. The first technique is based on order statistics that makes use of Wilks' formula. The second technique is based on a generalized additive model (GAM) that substitutes the thermal-hydraulic code, without and with consideration of errors in adjusting the GAM model. The comparison of the uncertainty analysis results makes use of several performance metrics such as coverage, coefficient of variation and conservativeness. Based on the results of these metrics it can be concluded that the GAMPE (GAM Plus Error) provides the best performance, in particular, when using small sample size, i.e. $n=59,93$. For larger sample sizes, i.e. $n=124,153$, GAMPE and Wilks' results presents similar performance.
\end{abstract}

Keywords: Uncertainty, PKL, Cladding Temperature, TRACE, Wilks, GAM, BEPU.

\footnotetext{
*Corresponding author

Email addresses: frasansa@etsii.upv.es (F. Sanchez-Saez), scarlos@iqn. upv.es (S. Carlos), jovillo0@iqn.upv.es (J.F. Villanueva), aisanche@eio.upv.es (A.I. Sanchez), smartore@iqn.upv.es (S Martorell)
} 


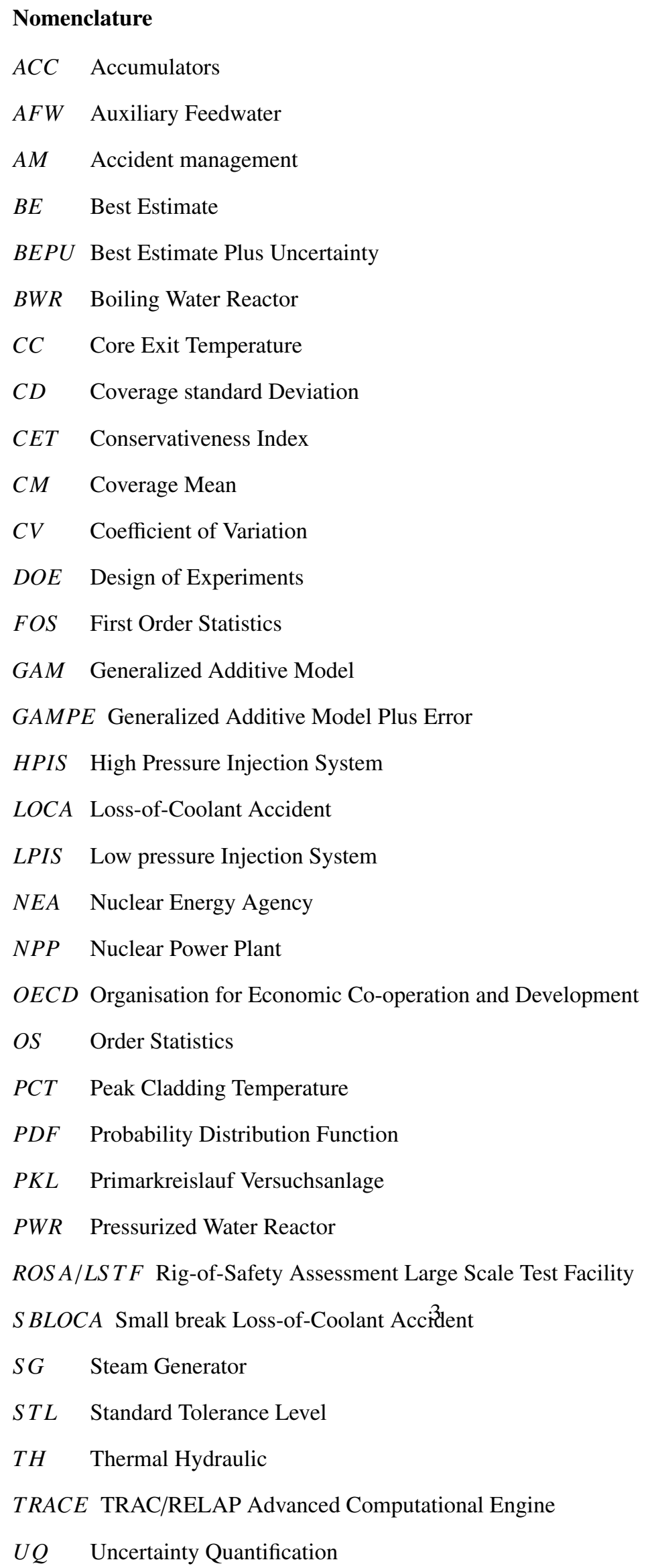




\section{Introduction}

Experimental facilities are of great importance in nuclear safety to improve the knowledge on commercial nuclear power plants (NPP) behavior under normal and accidental situations. Thus, it is possible to anticipate the evolution of the main safety variables under an accidental situation and identify generic issues that may affect the safety of nuclear power plants.

The results obtained in the experiments undertaken in such facilities are essential to develop and improve the models implemented in the thermal-hydraulic codes. The data collected in the experiments are necessary in the assessment of the capabilities of thermal-hydraulic codes to reproduce the different physical phenomena that may take place inside the reactor in accidental situations. Best estimate (BE) thermal-hydraulic (TH) codes, as RELAP-5 (Carlos et al., 2008), TRAC, CATHARE (Valette et al., 2011), ATHLET (Di Marcello et al., 2016) or TRACE (Belaid et al., 2013) are some examples. Nowadays, TRACE code (TRAC/RELAP Advanced Computational Engine) is being developed and validated to make use of the most favorable characteristics of RELAP-5 and TRAC codes to simulate both, Pressurized Water Reactor (PWR) and Boiling Water Reactor (BWR), technologies.

In the simulation process there exist code parameters and models that may be unknown or uncertain. This uncertainty may result in differences when a certain transient is simulated with a TH code depending on the code inputs chosen by the analyst (NAP, 2012).

Therefore, it is necessary to identify the most influencing parameters and models and to characterize their impact on the code response uncertainty, in terms of Figures of Merit (FOMs) related with safety variables. Often, a probabilistic approach is used to formulate the uncertainty of input parameters and data, and to propagate them towards the output using the BE code, which is also referred as a BEPU (Best Estimate Plus Uncertainty) approach. The purpose of the application of uncertainty propagation methodologies is to obtain the probability distribution function (PDF) of the safety related FOMs, or at least, an estimator, as the 95/95 Standard Tolerance Level (STL) (IAEA, 2009).

One of the most employed technique to obtain estimators of PDF output variables, i.e. FOMs, is OS (Order Statistics) based on Wilks' formula (Wilks , 1941). This technique permits to obtain the STL with a determined coverage/confidence value with reduced number of simulations (Beal, 2012), but only provides the safety variable bound. Other techniques, such as, the construction of surrogate models, or metamodelling, in which the TH code is substituted by a model in order to obtain an estimate of the FOMs of interest, or a related variable, permits extracting more information from the PDF with a reasonable computational cost (Carlos et al., 2013) (Di Maio et al., 2015) (Di Maio et al., 2016). However, as the number of input parameters increase, the construction of a metamodel requires a larger number of TH code simulations. In addition, NPP transient analysis involve simulations of complex phenomena, which can increase the computational burden. In fact, computational effort is one of the main problems in NPP transients simulations including uncertainty analysis, i.e. BEPU, as each TH code transient simulation may have a high computational cost. Thus, it is necessary to reduce the initially proposed uncertainty parameters in order to make the study feasible.

In this work, uncertainty analysis of a Small Break Loss of Coolant Accident (SBLOCA) at the PKL Test Facility is performed by simulation using two techniques: a metamodel based in Generalized Additive Models (GAM) and an OS using Wilks' formula.

The paper is organized as follows. In Section 2, the proposed methodology is explained, which includes the Plackett-Burman design of experiment, the Wilks' method and the employed metamodel. In addition, performance metrics are also described, which are used in order to check 
the goodness of the methods. Section 3 presents the case of application, which includes: a brief description of PKL facility, the TRACE model developed to simulate the transient, a description of the experiment SBLOCA, the base case results and the uncertainty analysis using the different techniques. Finally, Section 4 presents the main conclusions obtained.

\section{Methodology}

The methodology applied in this work is shown in Fig. 1 and consists of the following steps. First, a calibration of the simulation model by means of a base case is performed using experimental data. In this case, the safety variable of interest is the Peak Cladding Temperature (PCT) and the maximum value along the transient, i.e. the PCTmax, is adopted as FOM. Then, a sensitivity analysis has been performed in order to select the most influencing code inputs for the selected FOM and transient. Once the inputs have been selected, the uncertainty analysis is performed by two different approaches: Wilks' method and metamodelling. The metamodel selected to substitute TRACE code in the uncertainty analysis is a GAM, which is applied both with and without a treatment of the error. Finally, performance metrics are used in order to assess the goodness of the different uncertainty analysis methods.

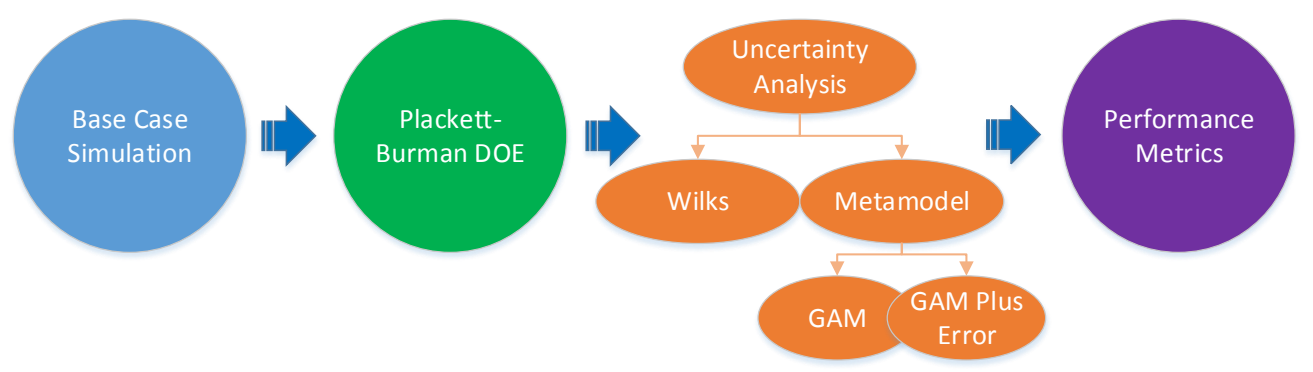

Figure 1: Methodology scheme.

\subsection{Design of Experiments. Plackett-Burman Design}

Design of experiments (DOE) objective is to plan experiments for extracting the maximum amount of information with the minimum number of tests or runs. In this study the objective of the DOE is to obtain the most significant variables, which can help in reducing the number of uncertain parameters. This will result in less runs to build the metamodel.

Among the different methods of DOE, the Plackett-Burman design, which objective is to find experimental designs for investigating the dependence of some dependent variables (outputs) on a number of independent variables (inputs) using a limited number of experiments (Plackett and Burman , 1946). This method belongs to the screening experimental designs methods, in which each variable takes L levels. Thus, if $k$ parameters are selected and, e.g. they have two levels, the minimum number of runs required by Plackett Burman design, is given by:

$$
P=4 * r
$$

Where $P$ is the total number of runs and $r$ is the lowest positive integer that verifies $P>k$. This is an advantage in terms of computational cost compared with the factorial design, where $L^{k}$ runs are required. In order to select which parameters are significant an hypothesis test based on analysis of variance (ANOVA) with the results of the Plackett-Burman DOE is performed. 


\subsection{Uncertainty Analysis techniques}

\subsubsection{Wilks' Method}

The Wilks' method consists of determining the number of calculations needed to verify compliance of the acceptance criteria with Standard Tolerance Levels (STL), typically 95/95 in accordance with current regulatory practice. Accordingly, the value of the safety variable that is compared with the corresponding acceptance criterion is often an upper or lower tolerance limit with level 95/95 instead of the output variable probability distribution. For example, it is often used one side tolerance interval of output variable based on the use of OS with STL=95/95. Using the well-known Wilks' formula, a First Order Statistics (FOS) requires a sample size of n=59 runs (Wilks , 1941).

$$
\beta=1-\gamma^{n}
$$

Where $n$ is the sample size required, $\beta$ is the probability that the interval $(-\infty, y(n)]$ covers a larger than $\gamma$ portion of the unknown distribution $G(y)$ of the output variable $y$, and $y(n)$ is the largest value of the sample.

FOS often provides conservative results, and several authors have explored the advantage of using OS of higher levels. However, the use of higher OS, which involves both an increase of the sample size and an increase in the confidence in the estimated percentile value implies a higher computational cost. (Beal, 2012) (Pal and Makai , 2005).

In this work, Wilks' method for first, second, third and fourth order are performed, which requires sample sizes of $n=59,93,124$ and 153, respectively. The number of samples required for maintain 95/95 STL in the different orders is obtained from the general Wilks' formula, shown in Eq. 3. Where $s=n+1-$ order number.

$$
\beta=\sum_{j=0}^{s-1}\left(\begin{array}{l}
n \\
j
\end{array}\right) \gamma^{j}(1-\gamma)^{n-j}
$$

One limitation of Wilks' method is that only provides the safety variable bound of the safety variable, and is necessary to use other methods for extracting additional information.

\subsubsection{Metamodel I. Generalized Additive Model (GAM)}

The substitution of the thermal-hydraulic code by metamodels is a widespread technique, which allows performing a larger number of runs with a reasonable computational cost. Some of the metamodels employed in nuclear safety analysis are, for example, finite mixture used in (Carlos et al., 2013) , (Di Maio et al., 2015), (Di Maio et al., 2016) for determining PCT. In this work, a GAM is proposed to undertake the uncertainty propagation analysis.

GAM is a natural extension of Generalized Linear Model (GLM), which has been commonly used to analyze the effect of different explanatory variables in output variables. The form of a GLM is given by:

$$
I(E(Y \mid X))=\eta(x)=\beta_{0}+\sum_{i=1}^{p} \beta_{i} x_{i}
$$

where $Y$ is the observed dependent response variable, $X$ are the observed independent covariates, $E$ is the expected value of $Y$ with known values of $X, \beta_{0}+\sum_{i=1}^{p} \beta_{i} x_{i}$ is a linear combination 
of unknown parameters $\beta$ and independent variables $x_{i}$ and $p$ is the number of independent variables. The link function $I$ is considered to be the same as a linear function of the predictors, $\eta(x)$. (Debon and Garcia-Diaz , 2012)

GLMs can present problems of bias and over-dispersion, therefore an alternative to these models are GAMs, introduced by (Hastie and Tibshirani , 1986), which are an extension of GLMs in the sense that they adjust nonparametric functions to study the relationship between predictive variables and the response. Non-parametric relationships between response and predictor variables are expressed in terms of smooth functions.

GAM can be obtained from the GLM by replacing the linear function in Eq. 4 with nonparametric, smooth functions, $s\left(x_{i}\right)$, as shown in Eq. 5:

$$
I(E(Y \mid X))=\eta(x)=s_{0}+\sum_{i=1}^{p} s_{i}\left(x_{i}\right)
$$

Thus, instead of a single coefficient for each variable in the model, in additive models an unspecified, non-parametric, function is estimated for each predictor, to achieve the best value of the dependent variable. This non-parametric function can be estimated in a flexible manner using cubic spline smoother, in back fitting algorithm. (Schimek and Turlach , 2000).

The main advantage of GAMs is that they can deal with non-linear and non-monotonic relationships between the response and the predictors without using variable transformations or polynomial terms, as GAM smoothing functions perform these tasks automatically. GAM is implemented in R language using the package mgcv (Wood , 2016). This package uses splines to create smooth functions, minimizing both the model deviance and the bias.

\subsubsection{Metamodel II. Generalized Additive Model Plus Error (GAMPE)}

The main drawback of GAM (and metamodels, in general) is that these techniques predicts the output in the mean of the distribution, so there are not conservatives. In order to fix this under-conservativeness of the GAM some strategies can be applied, such as multiply the output parameter by a safety factor, sum it a safety constant or adding the model error (Picheny ,2008). In this work an Error Distribution (ED) has been added to the output in order to achieve the required safety conservativeness. The ED is constructed by means of the error function, which is the difference between the value predicted $\left(Y_{\text {fitted }}\right)$ and the real output $\left(Y_{\text {Real }}\right)$ for the $\mathrm{n}$ points. Therefore, the ED with a conservative level of $(1-\alpha)$ is the $(1-\alpha)$ percentile of the error function distribution:

$$
E D=F^{-1}\left(1-\alpha ; Y_{\text {fitted }}-Y_{\text {Real }}\right)
$$

Where $F^{-1}$ is the inverse cumulative distribution function of the error distribution, and $\alpha$ is the significance level. Thus, the GAMPE (GAM Plus Error) model is constructed for each 95/95 STL estimation adding to each GAM model their associated ED.

\subsection{Performance Metrics}

In nuclear safety analysis is important to be conservative in order to assure the regulator mandatories. Therefore, in addition to the "classical" statistics it is necessary provide some metrics to evaluate the goodness of the models employed. In this work, the metrics extracted from reference (Picheny , 2008) are employed. First, the coverage $\left(C_{j}\right)$, which is the part of the reference distribution covered for each $j$ case is elaborated using the following expression: 


$$
C_{j}=\frac{\sum_{i=1}^{Z_{1}} I\left[\left(y_{i}\right) \leq(S T L(j))\right]}{Z_{1}}
$$

Where $Z_{1}$ is the sample size of the reference distribution, $y_{i}$ is the FOM value for each $i$ case of the reference distribution, $\operatorname{STL}(j)$ is the STL $95 / 95$ for each $\mathrm{j}$ case $(j=1, \ldots, N)$, and $I[\gamma]$ is the indicator function, which equals 1 if $\gamma$ is true and 0 if $\gamma$ is false.

Since $C_{j}$ is evaluated for $\mathrm{N}$ repetitions, the Coverage Mean (CM) of the individual coverages as a measure of coverage performance of the method, the Coverage standard Deviation (CD), as a measure of variability of the results, and the Coefficient of Variation (CV) are calculated. The last one, is the Coverage standard Deviation divided by the Coverage Mean in percent value as is expressed in eq. 8 .

$$
C V=\frac{C D}{C M} \cdot 100
$$

Finally, the conservativeness ( $\%$ of conservative predictions), which is the amount of cases out the total that are conservative, is calculated using:

$$
C C=\frac{\sum_{j=1}^{N} I[(S T L(j)) \geq(S V)]}{N}
$$

where, $\mathrm{SV}$ is the reference safety value and $\mathrm{N}$ are the number of repetitions used to calculate STL 95/95 in each uncertainty method.

\section{Case study}

In this work, the application case of the uncertainty analysis is a SBLOCA at the PKL Test Facility. The Nuclear Energy Agency (NEA), in the nuclear safety work area, auspices several programs that involve experiments in different experimental facilities (Carlos et al., 2011), (Reventos et al., 2008). Among them, one can find the OECD/NEA PKL-III project. The programme was mainly focused on investigating safety issues related to relevant and complex heat transfer mechanisms in current PWR plants as well as for new PWR design concepts (Jonnet et al., 2013). In this programme, the experiments were undertaken at Primarkreislauf Versuchsanlage facility (PKL) in Germany, which is a full-height, reduced-pressure scaled model of Phillisburg-2 Konvoi PWR design (Umminger et al., 2012), (Umminger et al., 2002).

\subsection{TRACE model of PKL facility}

The facility was modeled using the TRACE-5 V4 thermal-hydraulic code. TRACE code is being developed to make use of the most favorable characteristics of RELAP-5 and TRAC codes for both PWR and BWR technologies. TRACE has been designed to perform best-estimate analyses of loss-of-coolant accidents (LOCAs), operational transients, and other accident scenarios in reactors PWRs and BWRs, and it can also model phenomena occurring in experimental facilities designed to simulate transients in reactor systems (USNRC, 2014a) (USNRC, 2014b). The TRACE PKL model developed is shown in Fig. 2, which represents a SNAP view of the primary and secondary circuit (Applied Programming Technology, 2012). 


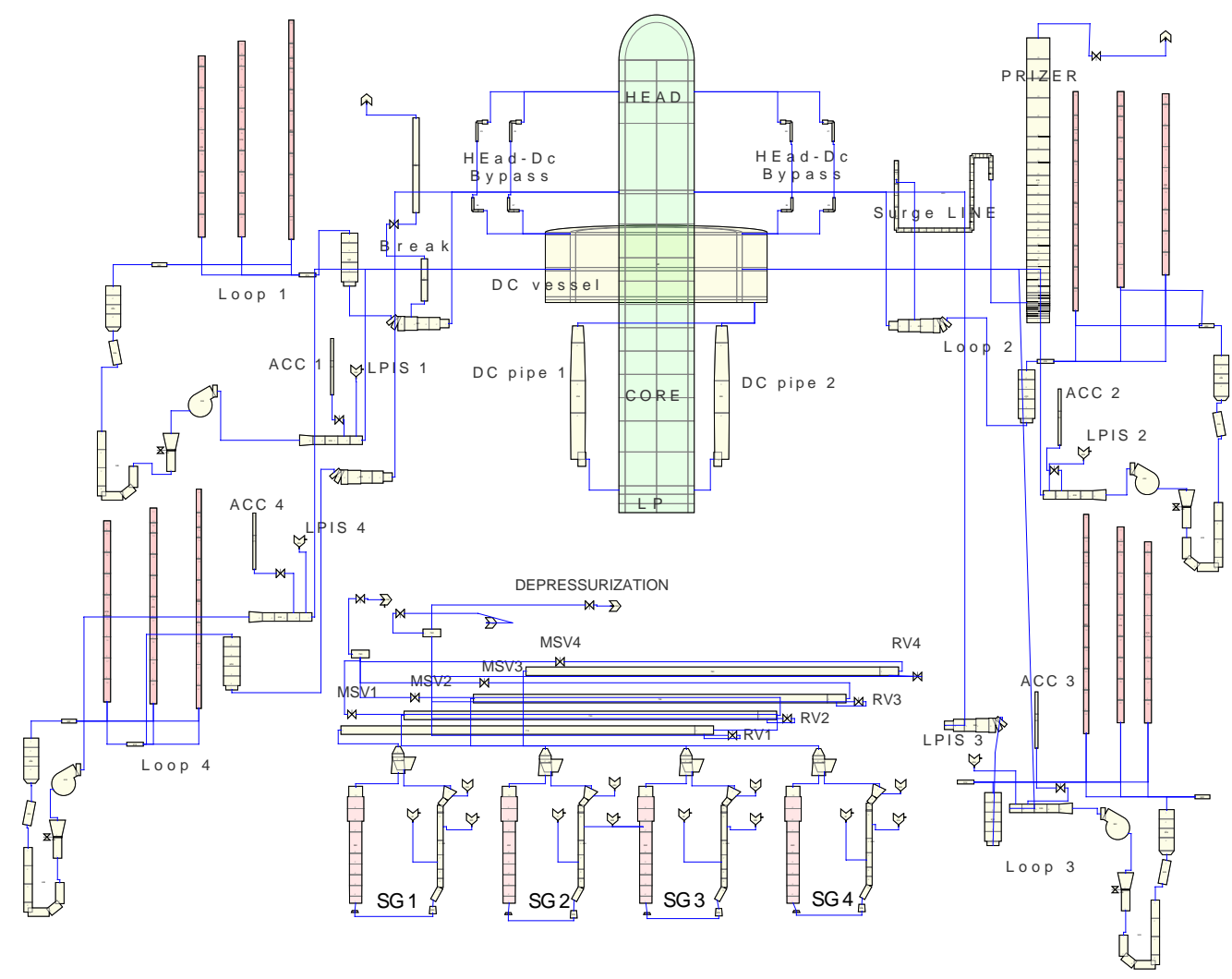

Figure 2: TRACE model for PKL primary circuit.

\subsection{Description of the experiment}

The experiment G7.1 performed at PKL facility consists of a SBLOCA in the hot leg of the primary side followed by a total failure of the high pressure injection system (HPIS) together with the failure of the automatic steam generators (SG) secondary-side cool-down, with a fixed power of $1.8 \%$ of the scaled nominal power of the reference plant. In such conditions, the accident mitigation procedure proposed is the manual depressurization of the SG secondary side, followed by the injection from the accumulators, and finally from the Low Pressure Injection System (LPIS). (Belaid et al., 2013) (Schoen et al., 2012). Table 1 presents the sequence of the events produced in PKL experiment.

Thus, the transient begins when the break is produced (at 500s) and the primary circuit empties. Secondary side depressurization is triggered by the core exit temperature (CET) set point at $623 \mathrm{~K}$. As all four SG are connected, the secondary side depressurization is homogeneous for all of them, and permits to reduce the primary pressure reaching the accumulators pressure set point. So this system injects water in the reactor coolant line and, later on, the primary pressure reaches LPIS activation set point. 
Table 1: Control sequence of major events in experiment.

\begin{tabular}{lll}
\hline Event & Condition & Time \\
\hline Break valve open & Start of the transient & $500 \mathrm{~s}$ \\
SG secondary-side depressurization & $C E T=623 \mathrm{~K}$ & $1800 \mathrm{~s}$ \\
Initiation of ACC system & $2.6 \mathrm{MPa}$ & $2000 \mathrm{~s}$ \\
Termination of ACC system & $1.0 \mathrm{MPa}$ & $2250 \mathrm{~s}$ \\
Initiation of LPI system & $0.8 M P a$ & $2500 \mathrm{~s}$ \\
\hline
\end{tabular}

\subsection{Simulation results. Base case}

In this transient, the most representative variables are CET and PCT, as they are the variables of interest to assure core integrity. Thus, while the PCT is the safety variable, the accident measure action proposed to mitigate the accident, i.e. the secondary side depressurization, is triggered by the CET value. In general, a good agreement between the experimental data and TRACE simulation of the temperatures is shown in Fig. 3, where it can be observed that the maximum PCT value (PCTmax) reached during the experiment is $816.2 \mathrm{~K}$.

At $2000 \mathrm{~s}$ the accumulators' injection is produced, and the consequent drop in the PCT is observed (see Fig. 3). PCT and CET evolutions are well reproduced by TRACE, although the CET is slightly higher in TRACE calculation than in the experimental data. In general, the plant behavior observed in the simulation agree with the experimental results, as explained in some more detail in reference (Carlos et al., 2016). So, this simulation is taken as base case to undertake the uncertainty analysis in the following sections, considering the PCTmax as output variable and adopting the methodology introduced in section 2 .

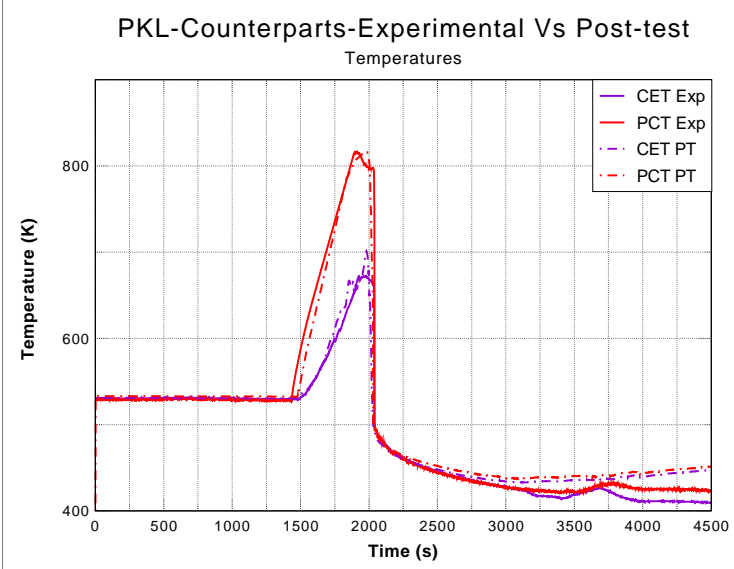

Figure 3: PCT and CET. 


\subsection{Selection of input parameters}

The next step of the uncertainty analysis methodology is to determine the parameters that are significant for the transient simulation in relation to the PCT evolution. It is necessary to account for the relevant physical phenomena associated with the transient analyzed. Some studies have been developed in order to establish a methodology to determine and rank the significant parameters. In these studies, the selection has been mainly developed using the information of the PIRT tables (Perez et al., 2011) (Kovtonyuk et al. , 2015) (Pourgol et al., 2016).

In this work, we make use of the uncertainty quantification (UQ) module implemented in the TRACE code (USNRC, 2014b). This module allows changing a list of parameters that normally are unseen or unchangeable from the input TRACE file to perform uncertainty quantification or sensitivity analysis. TRACE UQ sensitivity coefficients are a bundle of different parameters for adjusting the semi-empirical equations of heat transfer for different regimes, inter-facial drag models and other physical equation coefficients. In Table 2, the complete list of sensitivity coefficients in the TRACE UQ module is presented. In addition, we have also included the initial power, the clad thermal conductivity and specific heat capacity, the liquid and vapor/two-phase choke flow $(\mathrm{CF})$ model coefficients and the Critical Heat Flux multiplier, which are changed directly from the input TRACE file. Thus, the complete list of variables to be considered in this study is shown in Table 3 together with their statistical distribution data. Some variables that have available information from previous works are defined as normal or as uniform PDFs on the basis of (Perez et al., 2011). The TRACE UQ sensitivity coefficients are defined all of them as uniform PDF between 0.9-1.1 range.

The parameters of Table 2 are not specific for any transient, so when analyzing a SBLOCA not all the parameters may have a significant effect on the code response. Moreover, the complete list of parameters shown in Table 3 comprises 42 variables, which is an excessive number of parameters to construct the metamodel. Hence, in this work a design of experiment based on the Plackett-Burman design is proposed to reduce the number of variables involved in the uncertainty quantification process. 
Table 2: Uncertainty Quantification Sensitivity Coefficients Implemented in TRACE

\begin{tabular}{|c|c|c|}
\hline ID & Mnemonic Name & Description \\
\hline 1000 & bubSlugLiqIntHTC & Liquid to interface bubbly-slug heat transfer \\
\hline 1001 & annMistLiqIntHTC & Liquid to interface annular-mist heat transfer coefficient \\
\hline 1002 & transLiqIntHTC & Liquid to interface transition heat transfer coefficient \\
\hline 1003 & stratLiqIntHTC & Liquid to interface stratified heat transfer coefficient \\
\hline 1004 & bubSlugVapIntHTC & Vapor to interface bubbly-slug heat transfer coefficient \\
\hline 1005 & annMistVapIntHTC & Vapor to interface annular-mist heat transfer coefficient \\
\hline 1006 & transVapIntHTC & Vapor to interface transition heat transfer coefficient \\
\hline 1007 & stratVapIntHTC & Vapor to interface stratified heat transfer coefficient \\
\hline 1008 & singlePhaseLiqWallHTC & Single phase liquid to wall heat transfer coefficient \\
\hline 1009 & singlePhaseVapWallHTC & Single phase vapor to wall heat transfer coefficient \\
\hline 1010 & filmTransBoilTMin & Film to transition boiling Tmin criterion temperature \\
\hline 1011 & dispFlowFilmBoilHTC & Dispersed flow film boiling heat transfer coefficient \\
\hline 1012 & subBoilHTC & Subcooled boiling heat transfer coefficient \\
\hline 1013 & nucBoilHTC & Nucleate boiling heat transfer coefficient \\
\hline 1014 & DNBCHF & Departure from nucleate boiling / critical heat flux \\
\hline 1015 & transBoilHTC & Transition boiling heat transfer coefficient \\
\hline 1016 & gapConductance & Gap conductance coefficient \\
\hline 1017 & fuelThermalCond & Fuel thermal conductivity \\
\hline 1018 & cladMWRX & Cladding metal-water reaction rate coefficient \\
\hline 1019 & fuelRodIntPress & Rod internal pressure coefficient \\
\hline 1020 & burstTemp & Burst temperature coefficient \\
\hline 1021 & burstStrain & Burst strain coefficient \\
\hline 1022 & wallDrag & Wall drag coefficient \\
\hline 1023 & formLoss & Form loss coefficient \\
\hline 1024 & bubblyIntDrag & Interfacial drag (bubbly) coefficient \\
\hline 1025 & dropletIntDrag & Interfacial drag (droplet) coefficient \\
\hline 1026 & bubSlugIntDragBundle & Interfacial drag (bubbly/slug Rod Bundle - Bestion) coefficient \\
\hline 1027 & bubSlugIntDragVessel & Interfacial drag (bubbly/slug Vessel) coefficient \\
\hline 1028 & annMistIntDragVessel & Interfacial drag (annular/mist Vessel) coefficient \\
\hline 1029 & dffbIntDrag & Interfacial drag (dispersed flow film boiling) coefficient \\
\hline 1030 & invSlugIntDrag & Interfacial drag (inverted slug flow) coefficient \\
\hline 1031 & invAnnIntDrag & Interfacial drag (inverted annular flow) coefficient \\
\hline 1032 & tempFlood & Flooding coefficient temperature coefficient \\
\hline 1033 & lengthFlood & Flooding coefficient length coefficient \\
\hline 1034 & invAnnVapWallHTC & Vapor to wall inverted annular heat transfer coefficient \\
\hline 1035 & invAnnLiqWallHTC & Liquid to wall inverted annular heat transfer coefficient \\
\hline
\end{tabular}


Table 3: Input Uncertainty Parameters.

\begin{tabular}{|c|c|c|c|c|}
\hline Normal Distributed Inputs & Mean & Std Dev & Nominal Value & Units \\
\hline Power & 1.00 & 0.01 & 0.46 & MW \\
\hline Clad Thermal Conductivity & 1.00 & 0.05 & Table & $\mathrm{W} / \mathrm{m} \mathrm{K}$ \\
\hline Clad Specific Heat Capacity & 1.00 & 0.01 & Table & $\mathrm{J} / \mathrm{kg} \mathrm{K}$ \\
\hline Uniform Distributed Inputs & Min & Max & Nominal Value & Units \\
\hline Liquid CF & 0.90 & 1.10 & 1.10 & $(-)$ \\
\hline Vapor/Two-Phase CF & 0.90 & 1.10 & 0.96 & $(-)$ \\
\hline Critical Heat Flux multiplier & 0.90 & 1.10 & 1.00 & $(-)$ \\
\hline bubSlugLiqIntHTC & 0.90 & 1.10 & 1.00 & $(-)$ \\
\hline annMistLiqIntHTC & 0.90 & 1.10 & 1.00 & $(-)$ \\
\hline transLiqIntHTC & 0.90 & 1.10 & 1.00 & $(-)$ \\
\hline stratLiqIntHTC & 0.90 & 1.10 & 1.00 & $(-)$ \\
\hline bubSlugVapIntHTC & 0.90 & 1.10 & 1.00 & $(-)$ \\
\hline annMistVapIntHTC & 0.90 & 1.10 & 1.00 & $(-)$ \\
\hline transVapIntHTC & 0.90 & 1.10 & 1.00 & $(-)$ \\
\hline stratVapIntHTC & 0.90 & 1.10 & 1.00 & $(-)$ \\
\hline singlePhaseLiqWallHTC & 0.90 & 1.10 & 1.00 & $(-)$ \\
\hline singlePhaseVapWallHTC & 0.90 & 1.10 & 1.00 & $(-)$ \\
\hline filmTransBoilTMin & 0.90 & 1.10 & 1.00 & $(-)$ \\
\hline dispFlowFilmBoilHTC & 0.90 & 1.10 & 1.00 & $(-)$ \\
\hline subBoilHTC & 0.90 & 1.10 & 1.00 & $(-)$ \\
\hline nucBoilHTC & 0.90 & 1.10 & 1.00 & $(-)$ \\
\hline DNBCHF & 0.90 & 1.10 & 1.00 & $(-)$ \\
\hline transBoilHTC & 0.90 & 1.10 & 1.00 & $(-)$ \\
\hline gapConductance & 0.90 & 1.10 & 1.00 & $(-)$ \\
\hline fuelThermalCond & 0.90 & 1.10 & 1.00 & $(-)$ \\
\hline cladMWRX & 0.90 & 1.10 & 1.00 & $(-)$ \\
\hline fuelRodIntPress & 0.90 & 1.10 & 1.00 & $(-)$ \\
\hline burstTemp & 0.90 & 1.10 & 1.00 & $(-)$ \\
\hline burstStrain & 0.90 & 1.10 & 1.00 & $(-)$ \\
\hline wallDrag & 0.90 & 1.10 & 1.00 & $(-)$ \\
\hline formLoss & 0.90 & 1.10 & 1.00 & $(-)$ \\
\hline bubblyIntDrag & 0.90 & 1.10 & 1.00 & $(-)$ \\
\hline dropletIntDrag & 0.90 & 1.10 & 1.00 & $(-)$ \\
\hline bubSlugIntDragBundle & 0.90 & 1.10 & 1.00 & $(-)$ \\
\hline bubSlugIntDragVessel & 0.90 & 1.10 & 1.00 & $(-)$ \\
\hline annMistIntDragVessel & 0.90 & 1.10 & 1.00 & $(-)$ \\
\hline dffbIntDrag & 0.90 & 1.10 & 1.00 & $(-)$ \\
\hline invSlugIntDrag & 0.90 & 1.10 & 1.00 & $(-)$ \\
\hline invAnnIntDrag & 0.90 & 1.10 & 1.00 & $(-)$ \\
\hline tempFlood & 0.90 & 1.10 & 1.00 & $(-)$ \\
\hline lengthFlood & 0.90 & 1.10 & 1.00 & $(-)$ \\
\hline invAnnVapWallHTC & 0.90 & 1.10 & 1.00 & $(-)$ \\
\hline invAnnLiqWallHTC & 0.90 & 1.10 & 1.00 & $(-)$ \\
\hline
\end{tabular}




\subsection{Identification of most significant input parameters}

The PCTmax has been chosen as FOM in this study, which is extracted from the PCT evolution output performed by the TRACE code. In order to evaluate which of the 42 initial parameters shown in Table 3 have a real impact in FOM, a Plackett-Burman DOE has been performed. In this study, 42 parameters and two levels for each parameter have been considered, so, following the Plackett-Burman method, the total number of runs needed to perform the importance analysis is 44 (see Eq.1).

The results of the significant variables obtained from Plackett-Burman design of experiments are listed in Table 4. Specifically, Total Effect, t-value and p-value from the ANOVA test are listed in this table. The total effect respect the FOM, which determines the significance of the variables, is calculated as follows.

$$
\text { Total Effect }=\frac{2\left[\sum R(H)-\sum R(L)\right]}{N}
$$

Where $R(H)$ and $R(L)$ are the output values when the parameter appears in the high level and the low level respectively, and $N$ is the total number of runs.

The t-value is a standardized value obtained from the total effect, which can be compared with the contrast value corresponding to the confidence of ANOVA test with $\alpha$ value of 0.05 for 30 degrees of freedom, which is 2.04 in this case. Therefore, the parameters with a t-Value greater than the contrast value in absolute value, are considered significant.

Finally, the p-value is the probability enclosed at the right tail of the Student's t-distribution for the aforementioned t-values and can be directly compared with the $\alpha$ value, being the parameters that obtain a p-value lower than 0.05 as significant.

As a result of the DOE, it has been obtained that the most influencing variable is the initial power, followed by the single-phase vapor to wall heat transfer coefficient. With this methodology the parameters to be considered uncertain have been reduced with a confidence level of $95 \%$, i.e. $1-\alpha$, from 42 to 13 , as shown in Table 4 . Table 5 shows the statistical distribution data of the significant parameters to be used in the uncertainty quantification process.

Table 4: Plackett Burman test results

\begin{tabular}{lccl}
\hline Name & Total Effect & t-value & p-value \\
\hline Power & -31.13 & -6.92 & 0.000 \\
singlePhaseVapWallHTC & -22.44 & -4.99 & 0.000 \\
Vapor/Two-Phase CF & 21.84 & 4.85 & 0.000 \\
nucBoilHTC & 20.70 & 4.60 & 0.000 \\
Clad Thermal Cond. & -18.46 & -4.10 & 0.000 \\
dffbIntDrag & -17.39 & -3.87 & 0.001 \\
wallDrag & 14.28 & 3.17 & 0.003 \\
bubSlugIntDragBundle & 14.16 & 3.15 & 0.004 \\
singlePhaseLiqWallHTC & -13.35 & -2.97 & 0.006 \\
burstStrain & -12.16 & -2.70 & 0.011 \\
invSlugIntDrag & -11.88 & -2.64 & 0.013 \\
bubSlugLiqIntHTC & 10.70 & 2.38 & 0.024 \\
annMistLiqIntHTC & 9.28 & 2.06 & 0.048 \\
\hline
\end{tabular}


Table 5: Input Uncertainty Selected Parameters.

\begin{tabular}{|c|c|c|c|c|c|}
\hline Normal distributed Inputs & Mean & Std Dev & Value & Units & Description \\
\hline Power & 1.00 & 0.01 & 0.46 & MW & $\begin{array}{l}\text { Residual Power of the } \\
\text { facility at the transient }\end{array}$ \\
\hline Clad Thermal Conductivity & 1.00 & 0.05 & Table & $J / k g K$ & Rods (Ni-Cr alloy) Conductivity \\
\hline Uniform distributed Inputs & Min & Max & Value & Units & Description \\
\hline Vapor/TwoPhaseCF & 0.90 & 1.10 & 0.96 & $(-)$ & $\begin{array}{l}\text { Vapor and two phase fluid } \\
\text { coefficient for choked flow }\end{array}$ \\
\hline bubSlugLiqIntHTC & 0.90 & 1.10 & 1.00 & $(-)$ & $\begin{array}{l}\text { Liquid to interface } \\
\text { bubbly-slug heat transfer }\end{array}$ \\
\hline annMistLiqIntHTC & 0.90 & 1.10 & 1.00 & $(-)$ & $\begin{array}{l}\text { Liquid to interface } \\
\text { annular-mist heat transfer }\end{array}$ \\
\hline singlePhaseLiqWallHTC & 0.90 & 1.10 & 1.00 & $(-)$ & $\begin{array}{l}\text { Single phase liquid to wall } \\
\text { heat transfer coefficient }\end{array}$ \\
\hline singlePhaseVapWallHTC & 0.90 & 1.10 & 1.00 & $(-)$ & $\begin{array}{l}\text { Single phase vapor to wall } \\
\text { heat transfer coefficient }\end{array}$ \\
\hline nucBoilHTC & 0.90 & 1.10 & 1.00 & $(-)$ & $\begin{array}{l}\text { Nucleate boiling heat } \\
\text { transfer coefficient }\end{array}$ \\
\hline burstStrain & 0.90 & 1.10 & 1.00 & $(-)$ & Burst strain coefficient \\
\hline wallDrag & 0.90 & 1.10 & 1.00 & $(-)$ & Wall drag coefficient \\
\hline bubSlugIntDragBundle & 0.90 & 1.10 & 1.00 & $(-)$ & $\begin{array}{l}\text { Interfacial drag (bubbly/slug } \\
\text { Rod Bundle-Bestion) coefficient }\end{array}$ \\
\hline dffbIntDrag & 0.90 & 1.10 & 1.00 & $(-)$ & $\begin{array}{l}\text { Interfacial drag (dispersed } \\
\text { flow film boiling) coefficient }\end{array}$ \\
\hline invSlugIntDrag & 0.90 & 1.10 & 1.00 & $(-)$ & $\begin{array}{l}\text { Interfacial drag (Inverted } \\
\text { slug flow) coefficient }\end{array}$ \\
\hline
\end{tabular}

\subsection{Uncertainty analysis: Wilks versus GAM methods}

Figure 4 shows an overview of the uncertainty analysis procedure. First, two groups of size $Z_{1}=1000$ and $Z_{2}=10000$ values of input parameters are sampled by means of crude Monte Carlo from the PDFs exposed in Table 5. These two groups of size $Z_{1}$ and $Z_{2}$ are used as input to the TRACE code $\left(Z_{1}\right)$ and GAM (and GAMPE) simulations $\left(Z_{2}\right)$. With the $Z_{1}$ runs of the transient performed by TRACE, the safety variable associated to the FOM, i.e. the PCT evolution, is extracted and the PCTmax value (the FOM) for each simulation is calculated. The PCT evolutions are shown in Fig. 5, where it is observed that all of them are similar in form, although different in wide and length for all the runs. 


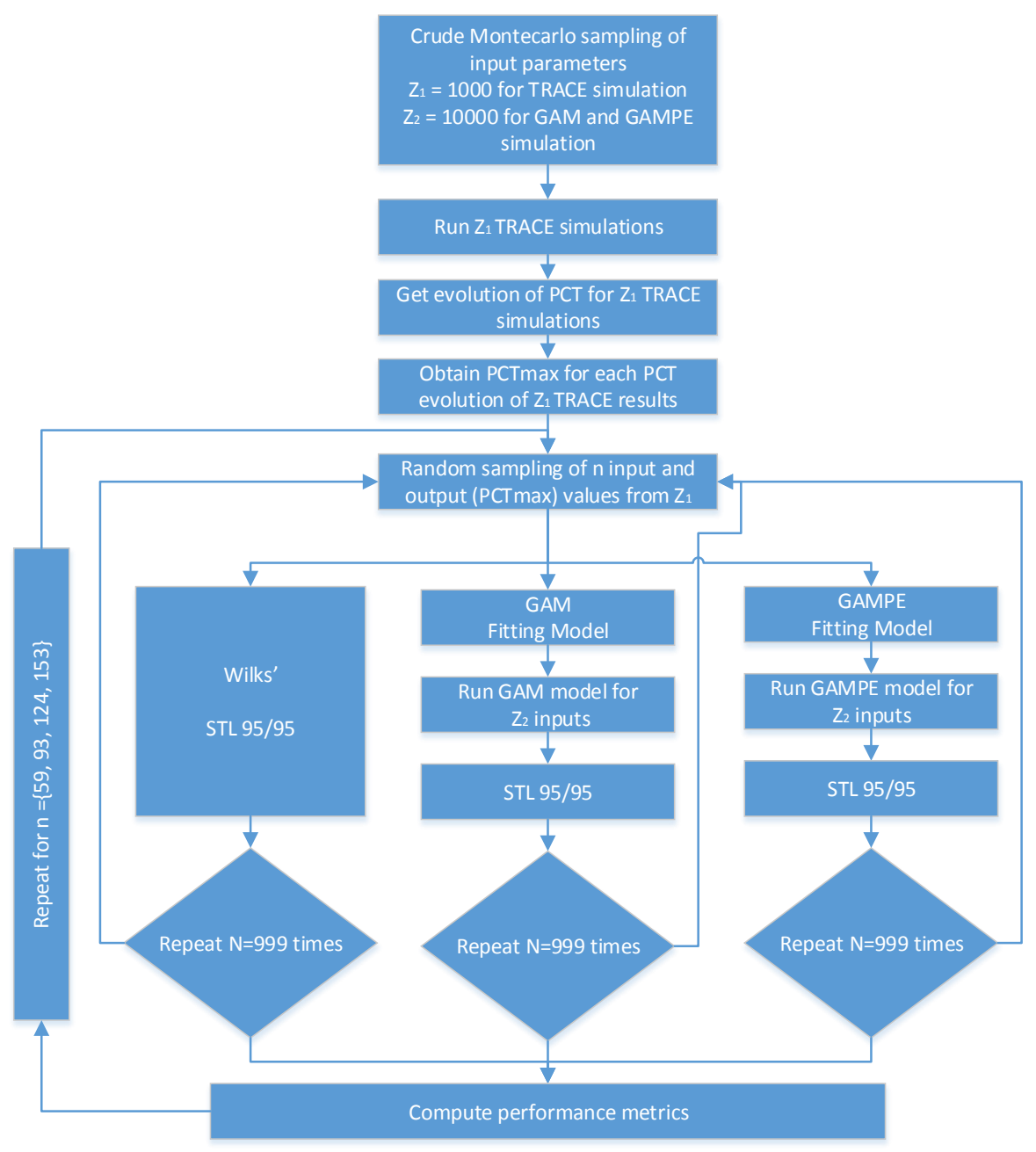

Figure 4: Uncertainty analysis scheme. 


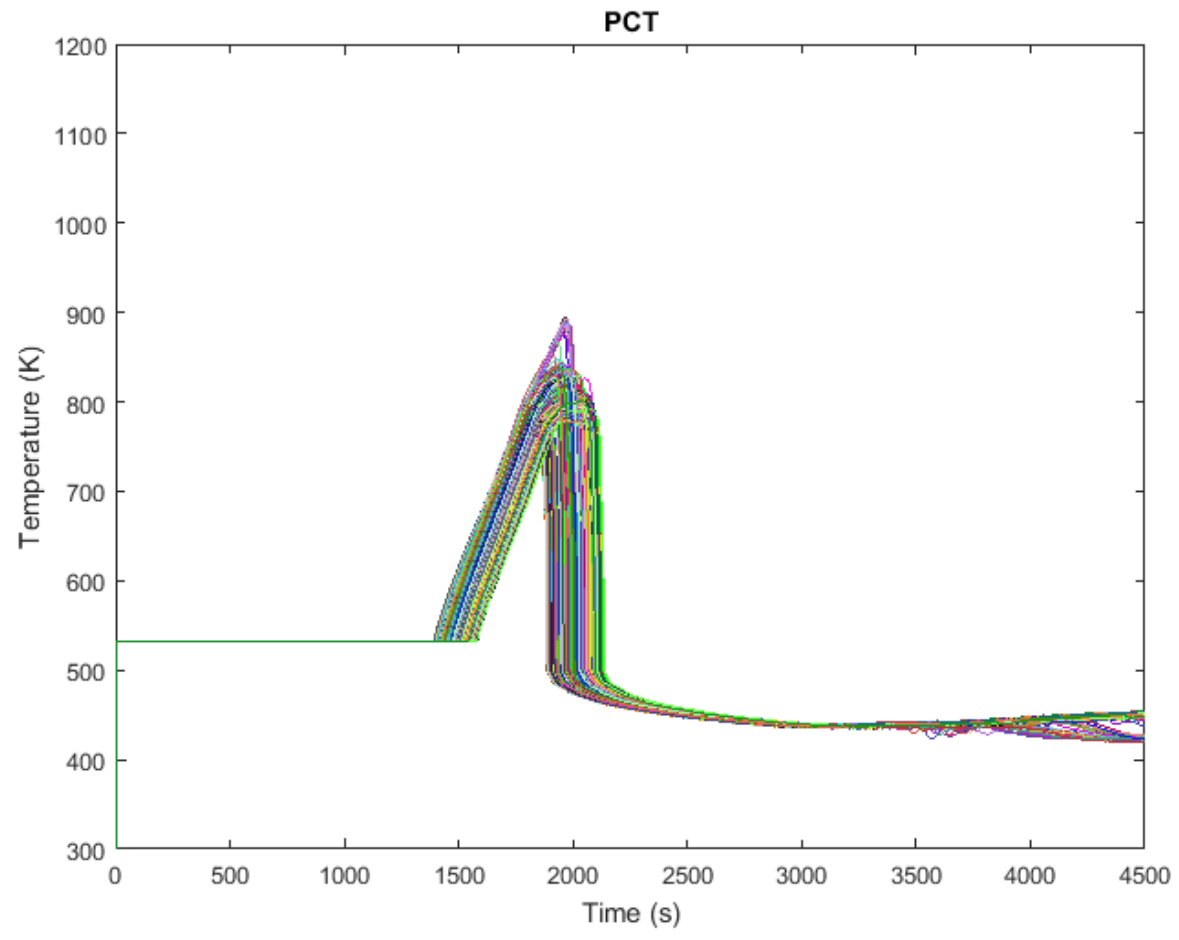

Figure 5: PCT TRACE results.

Then, a subset of $n$ samples is taken randomly from the $Z_{1}=1000$ inputs and the corresponding PCTmax results outputs results from TRACE code. This process is repeated for $N=999$ times. The uncertainty analysis is developed and the performance metrics are calculated for several sizes of $n=\{59,93,124,153\}$. The results are shown in Table 6 and represented graphically in Fig. 6.

Fig. 6 shows the PCTmax $(816.2 \mathrm{~K})$ corresponding to the Base Case (Section 3.3) and a PCTmax value taken as a reference $(835.8 \mathrm{~K})$, which corresponds to the 95 -percentile of the PCTmax distribution found using the results of the $Z_{1}=1000$ simulations. It also shows the distribution of the STL 95/95 results obtained from the $N=999$ repetitions for the corresponding $n$ for Wilks, GAM and GAMPE methods, which have been obtained as described hereafter.

For Wilks' method, the 95/95 STL is obtained simply ordering from highest to lowest PCTmax of the different subsets, and selecting the corresponding OS. Size $n=59$ is used to obtain the one-sided tolerance interval corresponding to the FOS, and the other $n$ sizes correspond to higher OS: second $(n=93)$, third $(n=124)$ and fourth $(n=153)$.

Since the procedure is repeated for $\mathrm{N}=999$ times, it can be estimated a mean value of the FOS with the Wilks' method of 863.2K. Moreover, a dispersion of the PCTmax values among the different repetitions is observed, which can be confirmed by the standard deviation of $23.6 \mathrm{~K}$ shown in Table 6.

The mean value of the second OS using Wilks' method of the $N=999$ samples is still conservative $(851.9 \mathrm{~K})$, which is around $10 \mathrm{~K}$ lower than FOS, and moreover, the dispersion is 
slightly reduced, although it is kept high yet (19.1K).

The third OS using Wilks' formula reduces both mean and standard deviation values to $845.6 \mathrm{~K}$ and $14.3 \mathrm{~K}$ respectively. This confirms that increasing the OS used in the estimation of $95 / 95$ STL, i.e. increasing the n-value, it is reached a value closer to the reference value with reduced dispersion.

Last, fourth OS obtained using Wilks' method results in a mean value of $842.4 \mathrm{~K}$ and the standard deviation is $10.5 \mathrm{~K}$, which are the best $95 / 95$ STL results, but at the expense of increasing the computational burden $(n=153)$.

For GAM surrogate models, the procedure for obtaining the 95/95 STL is the following. First, the GAM is constructed with R package and mgcv library as discussed in the Section 2 with the array formed for the input and output data of each subset of size $n$ sampled previously. The GAM model is built with the purpose of replacing TRACE code for a faster surrogate model, so, the PCTmax results of the different GAMs are obtained running these GAMs with $Z_{2}=10000$ input parameters sampled. The GAM surrogate models permit to obtain $Z_{2}=10000$ outputs quickly, since they are very low computer demanding. Finally, the outputs are ordered from highest to lowest and the 464th OS is selected, which is the value obtained from Eq. 3 (See Section 2.2.1) that guarantee a probability $\beta=0.95$ of covering at least $\gamma=0.95$ of the unknown output distribution for a sample size $n=10000$.

In order to assess the goodness of the metamodels obtained, the results of the GAM models are compared with the results obtained with the Wilks' method as it is shown in Table 6.

The behavior of the GAM models is similar to Wilks' results. Thus, when $n$ increases, the dispersion among the output values of the $N=999$ repetitions and the mean of these output values decreases. Moreover, GAM presents a lower dispersion among samples than Wilks' results for the same sample size $n$. However, GAM results are too close to the reference value, with a mean values of PCTmax between $835-840 \mathrm{~K}$, which indicates that there are non-conservative results in some cases, which can be an issue in nuclear safety analysis.

In order to avoid the lack of conservativeness of the original GAM models, the GAMPE explained in Section 2 is developed and used in a similar way as for the GAM. The results are shown in Fig. 6 and in Table 6. It can be seen that the coverage of GAMPE results in comparison with the reference value is near to $100 \%$. In addition, the GAMPE mean values are around $850 \mathrm{~K}$ no matter the $n$ size and present a lower standard deviation values than Wilks' results for the same sample size $n$. 
Table 6: PCTmax STL 95/95 estimation Results.

\begin{tabular}{lcccc}
\hline Method & Mean & Std Dev & Maximum & Minimum \\
\hline 1st Order Wilks & 863.2 & 23.6 & 893.8 & 833.8 \\
2nd Order Wilks & 851.9 & 19.1 & 892.6 & 833.3 \\
3rd Order Wilks & 845.6 & 14.3 & 889.7 & 834.2 \\
4th Order Wilks & 842.4 & 10.5 & 889.5 & 833.8 \\
GAM1 n=59 & 838.9 & 8.0 & 925.4 & 822.1 \\
GAM2 n=93 & 836.0 & 4.8 & 866.1 & 826.4 \\
GAM3 n=124 & 835.6 & 4.1 & 858.1 & 826.3 \\
GAM4 n=153 & 834.7 & 3.3 & 850.9 & 826.4 \\
GAMPE1 n=59 & 851.6 & 8.7 & 939.2 & 834.5 \\
GAMPE2 n=93 & 850.5 & 5.3 & 874.5 & 837.9 \\
GAMPE3 n=124 & 850.9 & 4.8 & 869.4 & 839.0 \\
GAMPE4 n=153 & 850.6 & 4.1 & 868.3 & 840.4 \\
\hline
\end{tabular}

Wilks' and GAMs - STL 95/95

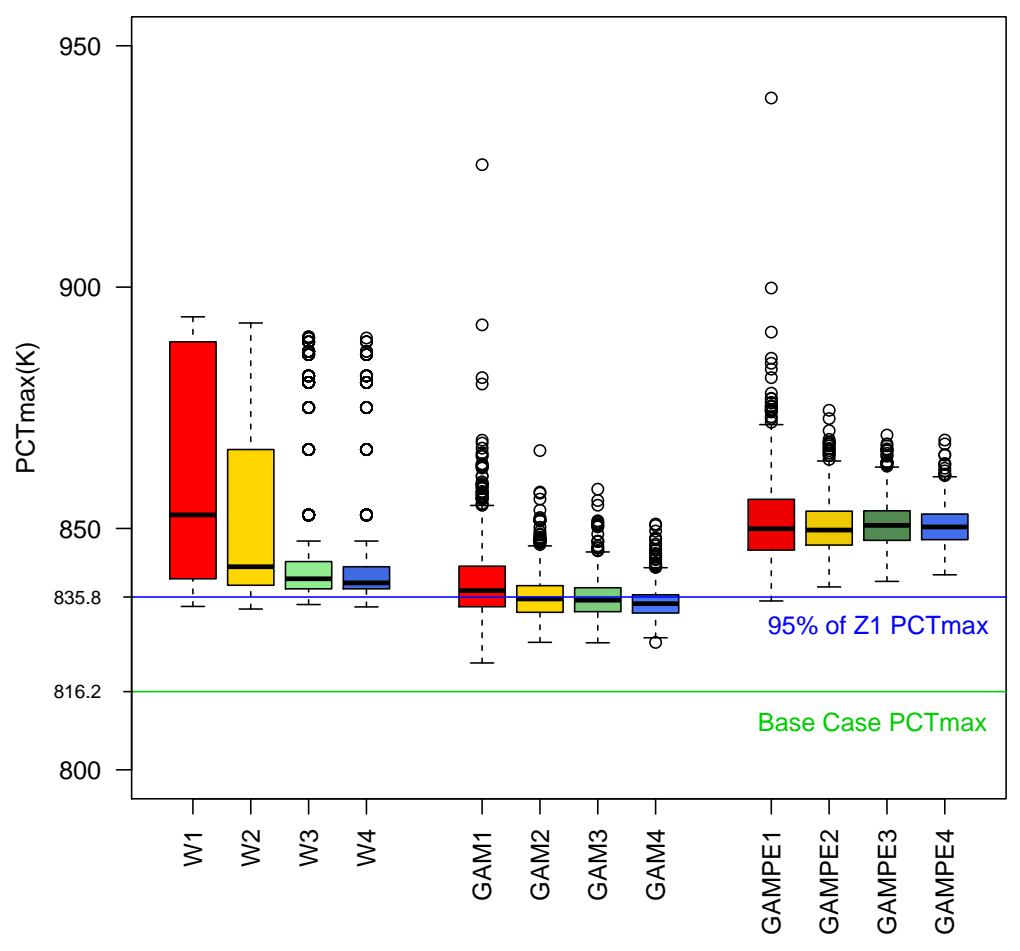

Figure 6: Box-Whisker diagram for Wilks and GAMs PCTmax STL 95/95 estimation. 


\subsection{Performance metrics. Comparation results}

The metrics used for assessing the goodness of the results of each method are shown in Table 7. Using CM and CC indexes, it can be observed that original GAM models obtain poor conservativeness values, whereas GAMPE have very good CC values of: $99.9 \%$ for $n=59$ and $100 \%$ for the other sizes. The Wilks' method, always provides conservative values for whatever $n$ size, with a high CC values, although slighty lower than GAMPE CC results. Regarding the CM values, the Wilks' method also presents similar values as GAMPE.

The dispersion of the results is measured with the CD index. For this metrics Table 7 shows a lower dispersion for GAMPE results as compared with both GAM and Wilks' results.

Finally, the robustness of the uncertainty analysis methods is measured with the CV index. Again GAMPE results are the best ones among the three methods applied, as the lower is the index, the greater is the robustness.

Therefore, one may conclude that the GAMPE provides the best results no matter the $n$ sample size, i.e. it provides the best results for the same computational cost as compared to Wilks' method. The benefit is even better for low sample sizes, $n=59,93$.

Table 7: Statistic Metrics.

\begin{tabular}{lcccc}
\hline $\begin{array}{l}\text { Uncertainty } \\
\text { Analysis Method }\end{array}$ & $\begin{array}{c}\text { Coverage } \\
\text { Mean (CM) }\end{array}$ & $\begin{array}{c}\text { Coverage } \\
\text { Std Dev. (CD) }\end{array}$ & $\begin{array}{c}\text { Coeff. } \\
\text { of Var. (CV) }\end{array}$ & $\begin{array}{c}\text { Conservative- } \\
\text { ness (CC) }\end{array}$ \\
\hline 1st Order Wilks & 98.4 & 1.44 & 1.46 & 96.8 \\
2nd Order Wilks & 97.9 & 1.34 & 1.37 & 95.9 \\
3rd Order Wilks & 97.6 & 1.27 & 1.30 & 96.0 \\
4th Order Wilks & 97.4 & 1.15 & 1.18 & 96.7 \\
GAM1 n=59 & 94.8 & 4.44 & 4.69 & 59.7 \\
GAM2 n=93 & 93.5 & 4.27 & 4.57 & 46.1 \\
GAM3 n=124 & 93.4 & 3.96 & 4.24 & 43.3 \\
GAM4 n=153 & 92.7 & 3.60 & 3.88 & 29.9 \\
GAMPE1 n=59 & 98.7 & 0.34 & 0.35 & 99.9 \\
GAMPE2 n=93 & 98.8 & 0.17 & 0.17 & 100.0 \\
GAMPE3 n=124 & 98.8 & 0.13 & 0.14 & 100.0 \\
GAMPE4 n=153 & 98.8 & 0.11 & 0.11 & 100.0 \\
\hline
\end{tabular}

\section{Conclusions}

A good agreement between the calculations predicted by TRACE for the behavior of the main safety variables and their respective experimental data is observed in the simulation of the base case corresponding to the PKL SBLOCA G7.1 experiment. However, in the simulation process uncertainties must be analyzed in order to measure their effect on safety variables of interest. This paper presents a methodology and the application to G7.1 experiment to perform the uncertainty analysis on the simulation of such experiment focusing on the PCTmax as FOM of interest.

First, the use of the Plackett-Burman method has demonstrated to reduce the amount of uncertain parameters to be considered. However, Plackett-Burman method does not addresses the effects of interactions among parameters. 
Next, the uncertainty analysis performed to estimate a tolerance interval of PCTmax attending to their uncertainties using Wilks' method shows conservative results and a great dispersion of the results depending on the sample sizes selected, i.e. the OS adopted. Increasing the OS based on the Wilks' formula reduces the estimated PCTmax, getting closer in average to the reference value corresponding to the 95th-percentile of the 1000 TRACE simulations (i.e. 835.8K) and the dispersion is reduced.

Alternatively, the GAM metamodel provides results, also in average, closer to the reference value, but it does not keep the conservatism for many of sample sizes, as the mean values for GAM outputs are below the aforementioned reference value (835.8K).

This problem has been solved by hybridizing the GAM model with an error function, which permits to obtain conservative results, in the same way as Wilks does, and at the same time the dispersion is reduced from two to three times the dispersion obtained with the same sample size using the Wilks' method. In addition, as it provides better results with the same computational cost since in average the mean values of the GAMPE are closer to the reference value, i.e. $835.8 \mathrm{~K}$.

Then, it may be concluded from the comparison of the results from the uncertainty analysis that the GAMPE provides the best overall performance, in particular, when using small sample size, i.e. $n=59,93$. For larger sample sizes, i.e. $n=124,153$, GAMPE and Wilks' results presents similar performance.

\section{Acknowledgments}

This work has been developed partially with the support of Programa de Apoyo a la Investigación y Desarrollo of UPV (PAID).

Authors are grateful to Spanish CSN (Consejo de Seguridad Nuclear) for the financial support of these researches: (Research Project SIN/4078/2013/640; MASA Project) and (Research Project STN/4524/2015/640; CAMP Project).

\section{References}

Applied Programming Technology, Inc., 2012. Symbolic Nuclear Analysis Package (SNAP). User's Manual. Version 2.2.1. Applied Programming Technology, Inc., Bloomsburg

Beal, D., 2012. Sample size determination for a nonparametric upper tolerance limit for any order statistic. In: Proceedings of the SESUG 2012. Paper SD-07, 1-8. SouthEast SAS Users Group, Durham, NC.

Belaid, S., Freixa J., Zerkak, O., 2010. Analysis of the Test OECD-PKL2 G7.1 with the Thermal-Hydraulic System Code TRACE. NUREG/IA-0432. U. S. Nuclear Regulatory Commission, Washington, DC.

Carlos, S., Villanueva, J.F., Martorell, S., Serradell, V., 2008. Analysis of a loss of residual heat removal system during mid-loop conditions at PKL facility using RELAP5/Mod3.3. Nucl. Eng. Des. 238 (10), $2561-2567$. http://doi.org/10.1016/j.nucengdes.2008.04.013

Carlos, S., Villanueva, JF., Martorell, S., Serradell V., 2011. Simulation of the F2.1 Experiment at PKL Facility Using RELAP5/MOD3. NUREG/IA-0250. U. S. Nuclear Regulatory Commission, Washington, DC.

Carlos, S., Sanchez, A., Ginestar, D., Martorell, S., 2013. Using finite mixture models in thermal-hydraulics system code uncertainty analysis. Nucl. Eng. Des. 262, 306-318. http://doi.org/10.1016/j.nucengdes.2013.04.030

Carlos, S., Querol, A., Gallardo, S., Sanchez-Saez, F., Villanueva, J. F., Martorell, S., Verd, G. 2016. Post-test analysis of the ROSA/LSTF and PKL counterpart test. Nucl. Eng. Des. 297, 81-94. http://doi.org/10.1016/j.nucengdes.2015.10.028

Debon, A., Garcia-Diaz J.C., 2012. Fault diagnosis and comparing risk for the steel coil manufacturing process using statistical models for binary data. Reliab. Eng. Syst. Safe. 100, 102-114. http://doi.org/10.1016/j.ress.2011.12.022

Di Maio, F., Nicola, G.,Zio, E. Yu, Y., 2015. Finite mixture models for sensitivity analysis of thermal hydraulic codes for passive safety systems analysis. Nucl. Eng. Des. 289, 144-154. http://doi.org/10.1016/j.nucengdes.2015.04.035 
Di Maio, F., Bandini, A., Zio, E., Alberola, S. C., Sanchez-Saez, F., Martorell, S., 2016. Bootstrapped-ensemble-based Sensitivity Analysis of a trace thermal-hydraulic model based on a limited number of PWR large break loca simulations. Reliab. Eng. Syst. Safe. 153, 122-134. http://doi.org/10.1016/j.ress.2016.04.013

Di Marcello, V., Imke, U., Sanchez, V., 2016. Validation and application of the system code ATHLET-CD for BWR severe accident analyses. Nucl. Eng. Des. 307, 284298. http://doi.org/10.1016/j.nucengdes.2016.07.013

Freixa, J., Martínez-Quiroga, V., Zerkak, O., Reventós, F., 2015. Modelling guidelines for core exit temperature simulations with system codes. Nucl. Eng. Des. 286, 116-129. http://doi.org/10.1016/j.nucengdes.2015.02.003

Glaeser, H., 2007. GRS Method for uncertainty and sensitivity evaluation of code results and applications. Sci. Technol. Nucl. Ins. 2008, 1-7. http://dx.doi.org/10.1155/2008/798901

Hastie, T., Tibshirani, R., 1986. Generalized Additive Models. Stat. Sci. 1 (3), 297-318.

IAEA, 2009. Deterministic Safety Analysis for Nuclear Power Plants. Specific Safety Guide No. SSG-2. International Atomic Energy Agency, Vienna.

Jonnet, J.R., Stempniewicz, M.M., de With, A., Wakker, P.H., 2013. RELAP5 analysis of PKL, main steam line break test. Nucl. Eng. Des. 265, 755-764. http://doi.org/10.1016/j.nucengdes.2013.09.010

Kovtonyuk, A., Petruzzi, A. DAuria, F., 2015. Post-BEMUSE Reflood Model Input Uncertainty Methods (PREMIUM) Benchmark Phase II: Identification of Influential Parameters. Nuclear Safety NEA/CSNI/R(2014)14. Nuclear Energy Agency.

Martinez-Quiroga, V., Reventos, F., Freixa, J., 2014. Applying UPC Scaling-Up Methodology to the LSTF-PKL Counterpart Test. Sci. Technol. Nucl. Ins. 2014, 1-18 . http://dx.doi.org/10.1155/2014/292916

NAP. 2012. Assessing the Reliability of Complex Models: Mathematical and Statistical Foundations of Verification, Validation, and Uncertainty Quantification. The National Academies Press, Washington, DC. https://doi.org/10.17226/13395

Pal, L., Makai, M., 2005. Statistical considerations on safety analysis. In: Proceedings of the International Meeting on Updates in Best Estimate Methods, Washington, DC.

Perez et al., 2011. Uncertainty and sensitivity analysis of a LBLOCA in a PWR Nuclear Power Plant: Results of the Phase V of the BEMUSE programme. Nucl. Eng. Des. 241 (10), 4206-4222. http://doi.org/10.1016/j.nucengdes.2011.08.019

Picheny, V., Kim, N., Haftka, T., Queipo, N.V., 2008. Conservative Predictions Using Surrogate Modeling. In: Proceedings of the 49th AIAA/ASME/ASCE/AHS/ASC Structures, Structural Dynamics, and Materials, Schaumburg, IL.

Plackett, R. L., Burman, J. P., 1946. The Design of Optimum Multifactorial Experiments. Biometrika. 33 (4), $305-325$ https://doi.org/10.1093/biomet/33.4.305

Plischke, E., 2009. An effective algorithm for computing global sensitivity indices (EASI). Reliab. Eng. Syst. Safe. 95 (4), 354-360. http://doi.org/10.1016/j.ress.2009.11.005

Pourgol-Mohamad, M., Hoseyni, S. Mohsen, Hoseyni, S. Mojtaba, Sepanloo, K., 2016. A practical sensitivity analysis method for ranking sources of uncertainty in thermalhydraulics applications. Nucl. Eng. Des. 305 (15), 400-410. http://doi.org/10.1016/j.nucengdes.2016.05.032

Reventos, F., Freixa, J., Batet, L., Pretel, C., Luebbesmeyer, D., Spaziani, D., Macek, J., Kasahara, F., Umminger, K., 2008. An analytical comparative exercise on the OECD-SETH PKL 2.2 experiment. Nucl. Eng. Des. 238 (4), 1146-1154. http://doi.org/10.1016/j.nucengdes.2007.10.002

Schimek, M. G., Turlach, B. A. Additive and Generalized Additive Models, in: Schimek, M. G. (Ed.), Smoothing and Regression: Approaches, Computation, and Application. John Wiley and Sons, Inc., Hoboken, NJ, pp. $277-327$. https://doi.org/10.1002/9781118150658.ch10

Schoen, B., Schollenberger, S. P., Umminger, K., 2012. Test PKL III G7.1: SB-LOCA with Total Failure of HPSI (Counterpart Testing with ROSA/LSTF) - Quick Look Report. Technical Report PTCTP-G/2011/en/0008, AREVA.

Toth, I., Prior, R., Sandervag, O., Umminger, K., Nakamura, H., Muellner, N., Cherubini, M., Del Nevo, A., D’Auria, F., Dreier, J., Alonso, J.R., Amri, A., 2010. Core Exit temperature (CET) in Accident Management of Nuclear Power Reactors. Nuclear Safety NEA/CSNI/R(2010)9. Nuclear Energy Agency.

Umminger, K., Mandl, R., Wegner, R., 2002. Restart of Natural circulation in a PWR-PKL test results and s-RELAP5 calculations. Nucl. Eng. Des. 215 (1-2), 39-50. http://doi.org/10.1016/S0029-5493(02)00040-7

Umminger, K., Dennhardt, L., Schollenberger, S., Schoen, B., 2011. Integral Test Facility PKL: Experimental PWR accident Investigation. Sci. Technol. Nucl. Ins. 2012, 1-15. http://dx.doi.org/10.1155/2012/891056

USNRC. 2014a. TRACE V5.840 Theory Manual Field Equations, Solution Methods, and Physical Models. U. S. Nuclear Regulatory Commission, Washington, DC

USNRC. 2014b. TRACE V5.840 User's Manual Volumes 1 and 2: Modeling Guidelines. U. S. Nuclear Regulatory Commission, Washington, DC.

Valette, M., Pouvreau, J., Bestion, D., Emonot, P., 2011. Revisiting large break LOCA with the CATHARE-3 three-field model. Nucl. Eng. Des. 241 (11), 4487-4496. http://doi.org/10.1016/j.nucengdes.2011.04.019

Wilks, S.S., 1941. Determination of sample sizes for setting tolerance limits. Ann. Math. Stat. 12 (1), 91-96. https://doi.org/10.1214/aoms/1177731788 
Wood, S., 2016. Mixed GAM Computation Vehicle with GCV/AIC/REML Smoothness Estimation. https://CRAN.Rproject. org $/$ package $=$ mgcv. 